\title{
PROPAGACIÓN DE UNA ONDA SOLITARIA EN CUERPOS DE AGUA SEMI-CONFINADOS
}

\author{
Susana Bastón, Maitane Olabarrieta, Pedro Lomónaco, Fernando J. Méndez, \\ Raúl Medina
}

Instituto de Hidráulica Ambiental "IH Cantabria", Universidad de Cantabria, España

bastons@unican.es

Resumen: La propagación de un tsunami hacia la costa y al interior de los estuarios se realiza, hoy en día, utilizando modelos numéricos hidrodinámicos de gran complejidad y elevado coste computacional. Sin embargo, en la costa hay cuerpos de agua semiconfinados (estuarios, sistemas playa-barrera-laguna, puertos, etc.), en los cuales se puede considerar que existe una única dirección de propagación del forzamiento exterior, y que por lo tanto, pueden ser estudiados utilizando un modelo hidrodinámico agregado de cajas. Estos modelos son ampliamente utilizados para conocer la propagación de la onda de marea pero también pueden resultar de utilidad para modelar fenómenos de naturaleza impulsiva como puede ser la llegada de un tsunami a la costa. Se ha desarrollado un modelo numérico de cajas, que ha sido validado con un ensayo en laboratorio. Se ha utilizado una onda solitaria para representar a un tsunami, y se ha comprobado que el modelo es capaz de reproducir el proceso de propagación de la onda solitaria al interior de un cuerpo de agua estrangulado. La conclusión del trabajo es que el modelo agregado permite, con poco esfuerzo computacional, evaluar los efectos de la propagación de una onda de tsunami a sistemas costeros.

\section{INTRODUCCIÓN}

La amenaza de los tsunamis, huracanes, erupciones volcánicas, terremotos y eventos extremos en general, es un tema que cada día preocupa más a la sociedad, por tratarse de sucesos asociados, generalmente, a los denominados 'desastres naturales'. Casi dos tercios de la población mundial vive en las zonas costeras, y de todos los riesgos naturales destructores que el hombre debe afrontar, los tsunamis son uno de los más devastadores, debido a la rapidez de propagación y a la violencia con la que el agua impacta en la costa.

Conocer el riesgo de inundación asociado a un potencial evento extremo es una información imprescindible para la ordenación territorial de la costa, el diseño de medidas de mitigación de catástrofes y la creación de planes de evacuación o planes de emergencia. Asimismo, en cumplimiento de la Directiva Europea relativa a la evaluación y gestión de los riesgos de inundación (2007/60/CE), tanto en España como en los demás países miembros de la unión, se hace necesario el desarrollo de herramientas sencillas para conocer en primera aproximación cuales son las áreas con un riesgo potencial de inundación significativo, es decir, aquellas zonas que tienen una alta probabilidad de ser inundadas. Para ello, es necesario disponer de modelos numéricos que permitan predecir cuál será la altura de ola, generada por un terremoto, en la costa.

Hoy en día, la propagación de un tsunami hacia la costa y al interior de los estuarios se realiza utilizando modelos numéricos hidrodinámicos de gran complejidad. Estos modelos requieren que la batimetría y la topografía del área de estudio tengan una gran resolución, de tal manera que para obtener resultados aceptables el tamaño de las celdas de la malla debe ser de unas decenas de metros o incluso menos en determinadas áreas. Normalmente, se utilizan mallas anidadas o embebidas, de forma que en aguas profundas el modelo resuelve una malla más espaciada y en las proximidades de la costa las mallas tienen mayor densidad de nodos. Por lo tanto, para aplicar estos modelos se requieren datos de topografía detallados y un importante esfuerzo computacional.

En la costa existen una gran cantidad de cuerpos de agua semi-estrangulados (estuarios, lagunas costeras, dársenas portuarias, etc.), que son susceptibles de verse afectados por un evento de tsunami. Independientemente del tamaño del cuerpo de agua (bahía o laguna), por definición, la conexión con el mar será estrecha y, por lo tanto, para que el modelo numérico tenga en cuenta su existencia, la resolución de la malla debe 
ser muy elevada. En estos sistemas costeros, en los que las dimensiones del cuerpo de agua son menores que la longitud de onda del forzamiento exterior del que depende su comportamiento hidrodinámico, se puede asumir que el flujo se propaga en una única dirección y que las variaciones de nivel son idénticas en todos los puntos (sistema cooscilante, (Defant, 1961)), de modo que se puede conocer su hidrodinámica utilizando un modelo numérico agregado de cajas.

Los modelos numéricos de cajas, basados en las ecuaciones unidimensionales de continuidad y de momento, solo necesitan unos pocos parámetros para definir la geometría del sistema (volumen, área, profundidad media, etc.). La gran ventaja de estos modelos es que se pueden variar fácilmente los parámetros que intervienen en el proceso que se está modelando, y así estudiar cuál es su influencia y formular reglas generales para describir la relación entre la geometría del sistema y la respuesta del forzamiento. Ejemplos de trabajos de análisis de sensibilidad se pueden encontrar en: van de Kreeke (1988, 1990), DiLorenzo (1988), Ridderinkhof (1988), Friedrichs y Madsen (1992) y Mass (1997), entre otros.

Se ha utilizado, en numerosas ocasiones, un modelo hidrodinámico agregado para describir el proceso de propagación de la onda de marea hacia el interior de un estuario (Speer y Aubrey, 1985; Friedrich y Aubrey, 1988; Stanev et al., 2003a, 2003b) pero no existe en la bibliografía la demostración de que estos modelos sean adecuados para conocer la respuesta de un evento de tsunami en el interior de un cuerpo de agua semiconfinado.

El objetivo principal de este trabajo es demostrar que un modelo numérico agregado es adecuado para resolver cuál es la respuesta en sistemas costeros ante la llegada de un tsunami. Para ello, se ha desarrollado un modelo numérico que ha sido validado con un ensayo en laboratorio, de tal forma que el artículo se divide en dos partes, en primer lugar se presentan las ecuaciones básicas del modelo numérico propuesto, basado en la teoría de bahías de Keulegan (Keulegan, 1951), así como las desarrolladas para geometrías más complejas. En la segunda parte se describe el ensayo de laboratorio realizado para validar el modelo y se presentan los resultados y la comparación de éstos con los que se obtienen del modelo numérico. La conclusión que se deriva de este trabajo es que el modelo agregado per- mite, con poco esfuerzo computacional, evaluar los efectos de la propagación de una onda de tsunami a sistemas costeros.

\section{PROPAGACIÓN DE ONDAS LARGAS EN SISTEMAS COSTEROS}

\section{Ecuaciones básicas del modelo agregado}

Las variaciones de nivel en un cuerpo de agua semiestrangulado (Figura 1) se describen mediante las ecuaciones de momento y continuidad:

$$
\begin{aligned}
\frac{d u}{d t} & =\frac{g}{L}\left(\eta_{o}-\eta_{b}-F \frac{u|u|}{2 g}\right) \\
\frac{d V}{d t} & =A_{c} \cdot u
\end{aligned}
$$

donde $g$ es la aceleración de la gravedad, $u$ es la velocidad a través del canal, $\eta_{b}$ la variación de nivel en la bahía y $\eta_{o}$ el nivel en el mar exterior, que es la evolución temporal de la superficie libre asociada a las variaciones del nivel del mar producidas por el tsunami. Las variables que describen las características geométricas del sistema son el área de la bahía $\left(A_{b}\right)$, el área de la sección transversal del canal $\left(A_{c}\right)$ y la longitud del mismo $(L)$.

$F$ es el parámetro de fricción definido por Bruun et al. (1978), que incluye la pérdida de carga debida a la contracción y expansión del flujo a la entrada y salida del canal, respectivamente, y la fricción a lo largo del canal:

$$
F=k_{\text {ent }}+k_{\text {sal }}+\frac{f L}{4 h_{c}}=K+\frac{f L}{4 h_{c}}
$$

donde $h$ es la profundidad del canal correspondiente al nivel medio. Las pérdidas de carga localizadas se engloban dentro de un único parámetro $K$, que es el que se utiliza en la validación y calibración del modelo numérico.

El área transversal del canal se obtiene con la siguiente expresión

$$
A_{c}=A_{c}^{o}\left(1+\eta_{c}\right)
$$

en la que $A_{c}^{o}=W_{c} \cdot h_{c}$, donde $W_{c}$ es la anchura del canal existente cuando el nivel coincide con el nivel medio. El nivel en el canal $\left(\eta_{c}\right)$ puede sufrir variaciones importantes y depende fundamentalmente del nivel en el exterior $\left(\eta_{o}\right)$. 

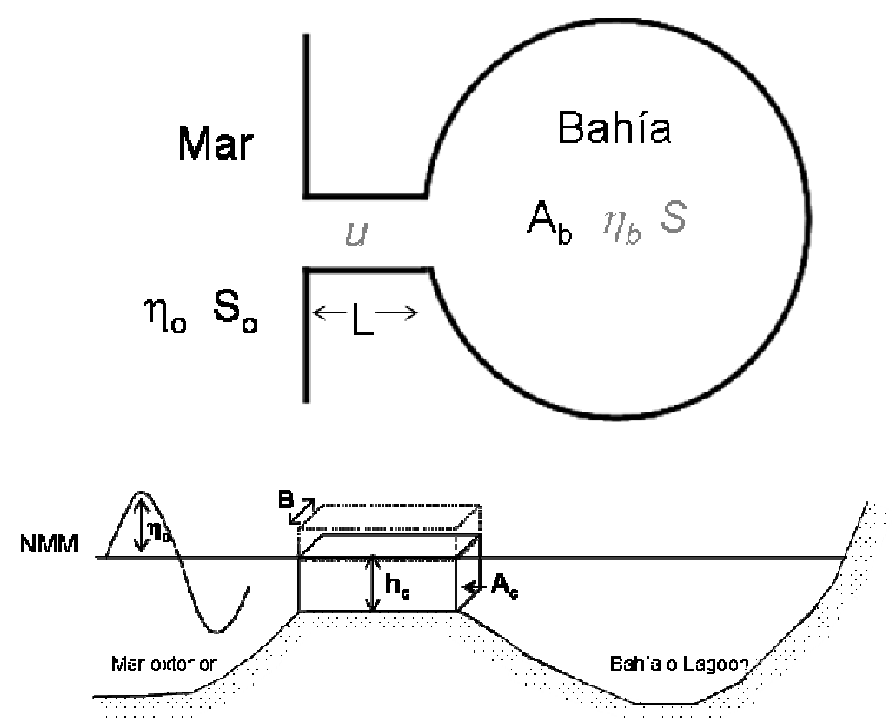

Figura 1. Esquema conceptual de un cuerpo de agua estrangulado, en el que se muestran las variables que necesita el modelo (oscuras) y las que resuelve (claras y en cursiva)

El volumen de agua asociado a la propagación de una onda larga hacia el interior de un sistema costero se define como:

$$
V=\int_{0}^{\eta}\left(A_{b}(z) \cdot d z\right.
$$

donde $A_{b}$ es el área de la bahía. Se asume que los cajeros del sistema son verticales, por tanto el valor del área de la bahía, $A_{b}$, es constante.

Si se asume que $V=A_{b} \cdot \eta_{b}$, el sistema de ecs.(1) y (2) se convierte en:

$$
\begin{aligned}
& \frac{d u}{d t}=\frac{g}{L}\left(\eta_{o}-\eta_{b}-F \frac{u|u|}{2 g}\right) \\
& \frac{d \eta}{d t}=\frac{A_{c}}{A_{b}} \cdot u
\end{aligned}
$$

resultando un sistema de ecuaciones diferenciales (6) y (7) con dos incógnitas ( $u$ y $\eta_{b}$ ), donde la respuesta del sistema dependerá del forzamiento exterior $\left(\eta_{o}(t)\right)$.

\section{Ejemplos teóricos de aplicación del modelo con una onda solitaria}

En la literatura, el forzamiento exterior, $\eta_{o}$, se describe, generalmente, mediante una función armónica para simular la onda de marea. Esto se debe a que es la onda de marea la que afecta de forma continua a los sistemas costeros y es la responsable directa de la morfología de equilibrio de los mismos. Sin embargo, el modelo puede resolver cuál es la respuesta, en un sistema costero, de una onda larga que no tiene porqué ser necesariamente la onda de marea.

En la Figura 2 se presenta la solución del modelo para un caso teórico, en el que se introduce el nivel en el mar exterior, $\eta_{o}$, mediante la expresión de una onda solitaria (8), para representar la evolución temporal de la superficie libre asociada a un tsunami

$$
\eta_{o}=H_{x} \sec h^{2}\left[\sqrt{\frac{3}{4} \frac{H_{x}}{h_{x}^{3}}}(c t)\right]
$$

donde $H_{x}$ es la altura de la onda en el exterior del cuerpo de agua sometido a estudio, $h_{x}$ la profundidad bajo el nivel medio del mar (NMM) existente en el exterior del sistema, $t$ el tiempo y $c$ la celeridad de la onda, que tiene la siguiente expresión:

$$
c=\sqrt{\frac{g h_{x}}{1-\left(h_{x} / h_{x}\right)}}
$$

La onda solitaria propuesta tiene $1 \mathrm{~m}$ de altura y el $95 \%$ de su volumen se comprende en un espacio temporal de $500 \mathrm{~s}$. Las características del sistema son: el área de la bahía $10^{7} \mathrm{~m}^{2}$, y un canal de $10 \mathrm{~m}$ de profundidad media y $1 \mathrm{Km}$ de anchura.

Se presentan nueve gráficas, en las tres primeras (fila superior) se muestra la influencia de la anchura del canal, en las tres siguientes (fila intermedia) la influencia de la longitud del canal y en las tres últimas (fila inferior) la influencia del área de la bahía. En los casos (1-3) la longitud del canal fijada es $100 \mathrm{~m}$ y la anchura es 10 $\mathrm{m}$ en la primera figura, $100 \mathrm{~m}$ en la segunda y $1000 \mathrm{~m}$ en la tercera. En el primer caso, como el paso de agua es tan angosto, el nivel en el interior de la bahía apenas sufre variación como consecuencia de la onda solitaria, pero a medida 

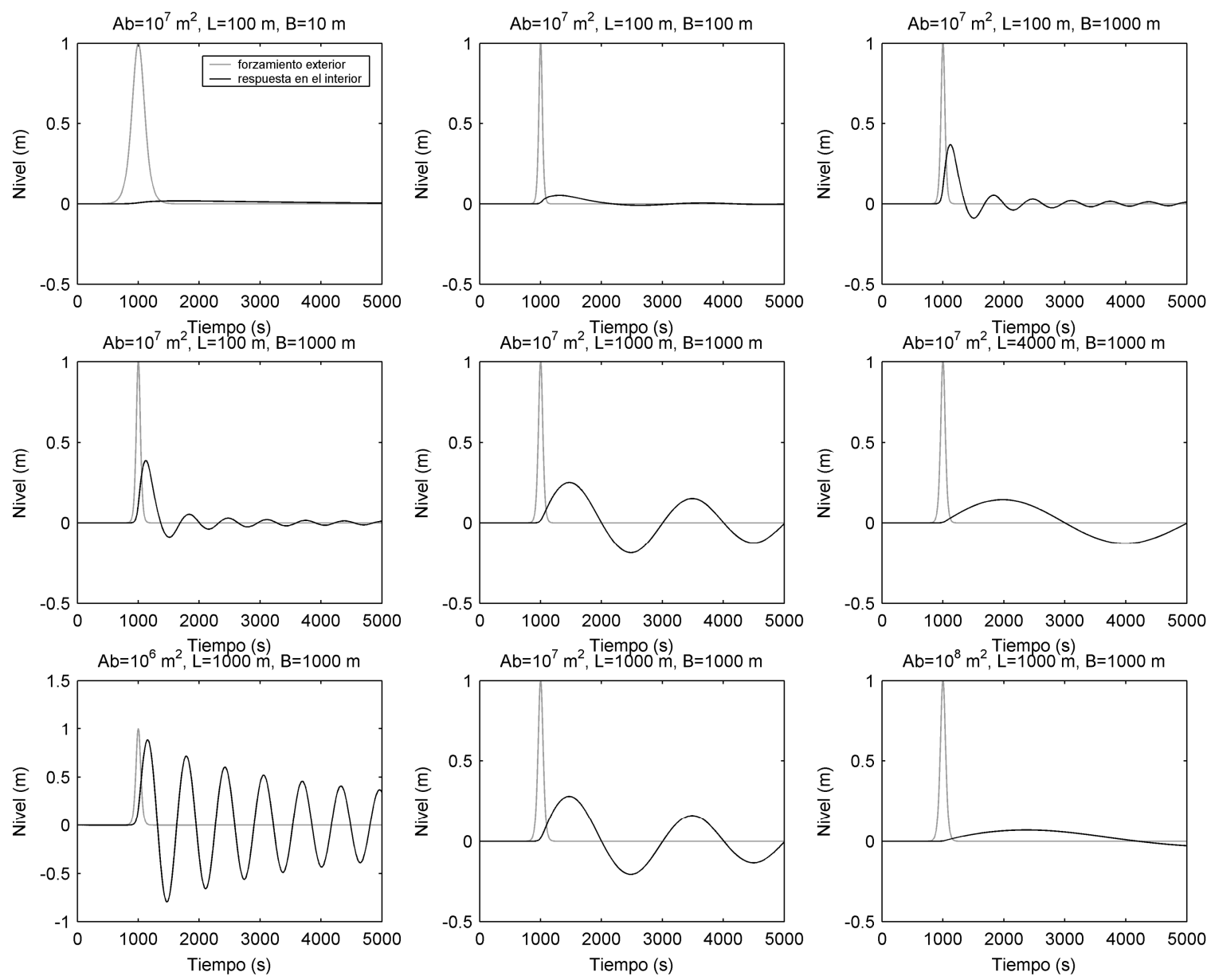

Figura 2. Ejemplos de variación del nivel en el interior de un sistema estuarino ante la existencia de una onda solitaria en el exterior

que aumenta la anchura del canal se puede ver como el pulso del exterior provoca un pulso en el interior del estuario, de mayor amplitud cuanto mayor sea la anchura del canal.

En los casos (4-6) la anchura del canal fijada es de $1 \mathrm{Km}$ y varía la longitud desde $100 \mathrm{~m}$ a $4 \mathrm{Km}$. Cuanto mayor es la longitud del canal la onda pierde energía por efecto de la fricción y la amplitud que se alcanza en el interior del sistema es menor.

En los casos (7-9) se fija la longitud del canal en $1 \mathrm{Km}$ y varía el área de la bahía un orden de magnitud. Destaca el efecto que se observa para el caso de $A_{b}=10^{6} \mathrm{~m}^{2}$, en el que la respuesta en el interior supera en amplitud al forzamiento exterior. Esto se debe a efectos de resonancia para esta frecuencia. También se puede ver cómo si el área de la bahía es un orden de magnitud mayor, el volumen de agua que entra en la bahía se tiene que repartir en una mayor superficie, de modo que la amplitud que alcanza la onda es menor.

\section{Aplicación a sistemas con geometrías más complejas}

En la realidad, los estuarios muchas veces presentan una geometría más compleja que una bahía comunicada con el mar por un estrecho y largo canal. Es frecuente encontrar en el litoral sistemas formados por más de un cuerpo de agua estrangulado, bien por causas naturales 0 como consecuencia de alteraciones antrópicas, como por ejemplo estuarios subdivididos por la creación de una carretera, represamientos, etc.

En beneficio de conocer la hidrodinámica de sistemas más complejos, Salles (2001) estudió la estabilidad de sistemas comunicados con el mar por más de un canal y Jain (2002) derivó las ecuaciones para resolver la hidrodinámica de sistemas complejos entre los que se pueden encontrar sistemas con tres bahías comunicadas con el mar exterior por un único canal. Pero no se han encontrado estudios en la literatura en la que estos modelos se hayan validado ni aplicado al estudio de la propagación de una onda de tsunami al interior de un sistema costero. 


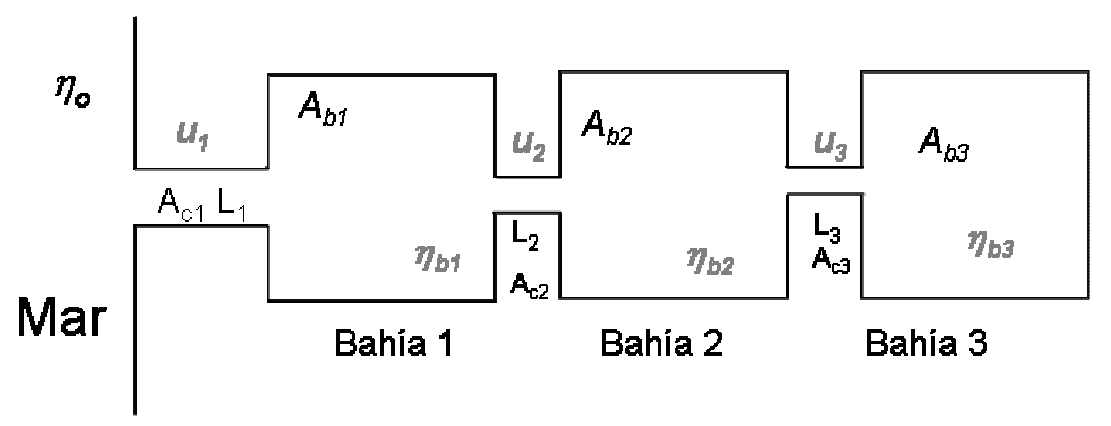

Figura 3. Esquema descriptivo de un sistema de tres bahías conectadas en serie. Se presentan las variables que intervienen, en negro los datos de entrada al modelo y en gris las variables de salida

\begin{tabular}{lccc}
\hline Características comunes & Bahía 1 & Bahía 2 & Bahía 3 \\
\hline Longitud del canal $(L),(\mathrm{m})$ & 100 & 100 & 100 \\
Profundidad del canal $\left(h_{c}\right),(\mathrm{m})$ & 10 & 10 & 10 \\
\hline Caso 1 & & & \\
Área de la Bahía $\left(A_{b}\right),\left(\mathrm{m}^{2}\right)$ & $10^{6}$ & $10^{6}$ & $10^{6}$ \\
Anchura del canal $(B),(\mathrm{m})$ & 200 & 100 & 50 \\
\hline Caso 2 & & & \\
Área de la Bahía $\left(A_{b}\right),\left(\mathrm{m}^{2}\right)$ & $10^{6}$ & $10^{6}$ & $10^{6}$ \\
Anchura del canal $(B),(\mathrm{m})$ & 20 & 10 & 5 \\
\hline Caso 3 & & & \\
Área de la Bahía $\left(A_{b}\right),\left(\mathrm{m}^{2}\right)$ & $10^{6}$ & $2 \cdot 10^{6}$ & $5 \cdot 10^{6}$ \\
Anchura del canal $(B),(\mathrm{m})$ & 200 & 100 & 50 \\
\hline
\end{tabular}

Tabla 1. Características que describen cada uno de los casos teóricos propuestos para sistemas con geometrías complejas

Se presentan, a continuación, las ecuaciones para un sistema formado por tres bahías parcialmente estranguladas conectadas en serie (Figura 3), por ser la geometría utilizada para validar el modelo en laboratorio, que se presenta en el siguiente apartado.

Ecuaciones de conservación del momento:

$$
\begin{aligned}
& \frac{d u_{1}}{d t}=\frac{g}{L_{1}}\left(\eta_{o}-\eta_{1}-F_{1} \frac{u_{1}\left|u_{1}\right|}{2 g}\right) \\
& \frac{d u_{2}}{d t}=\frac{g}{L_{2}}\left(\eta_{1}-\eta_{2}-F_{2} \frac{u_{2}\left|u_{2}\right|}{2 g}\right) \\
& \frac{d u_{3}}{d t}=\frac{g}{L_{3}}\left(\eta_{2}-\eta_{3}-F_{3} \frac{u_{3}\left|u_{3}\right|}{2 g}\right)
\end{aligned}
$$

La velocidad del flujo en cada uno de los canales depende del nivel que existe en los dos cuerpos de agua que comunica y de la fricción a la que se ve sometido.
Ecuaciones de conservación de la masa:

$$
\begin{aligned}
\frac{d \eta_{1}}{d t} & =\frac{A_{c 1}}{A_{b 1}} u_{1}+\frac{A_{c 2}}{A_{b 1}} u_{2} \\
\frac{d \eta_{2}}{d t} & =\frac{A_{c 2}}{A_{b 2}} u_{2}+\frac{A_{c 3}}{A_{b 2}} u_{3} \\
\frac{d \eta_{3}}{d t} & =\frac{A_{c 3}}{A_{b 3}} u_{3}
\end{aligned}
$$

El nivel en la bahía más alejada del mar (bahía 3 ) sólo depende del flujo que pasa por el canal que la comunica con el elemento anterior, y el de las bahías intermedias (bahías 1 y 2 ) depende del flujo que intercambian con los cuerpos de agua anterior y posterior.

El modelo numérico es capaz de obtener la respuesta de un sistema con geometría compleja ante la llegada de un tsunami. En la Figura 4 se presentan las soluciones obtenidas para tres casos teóricos, cuyas características geométricas se recogen en la Tabla 1. 

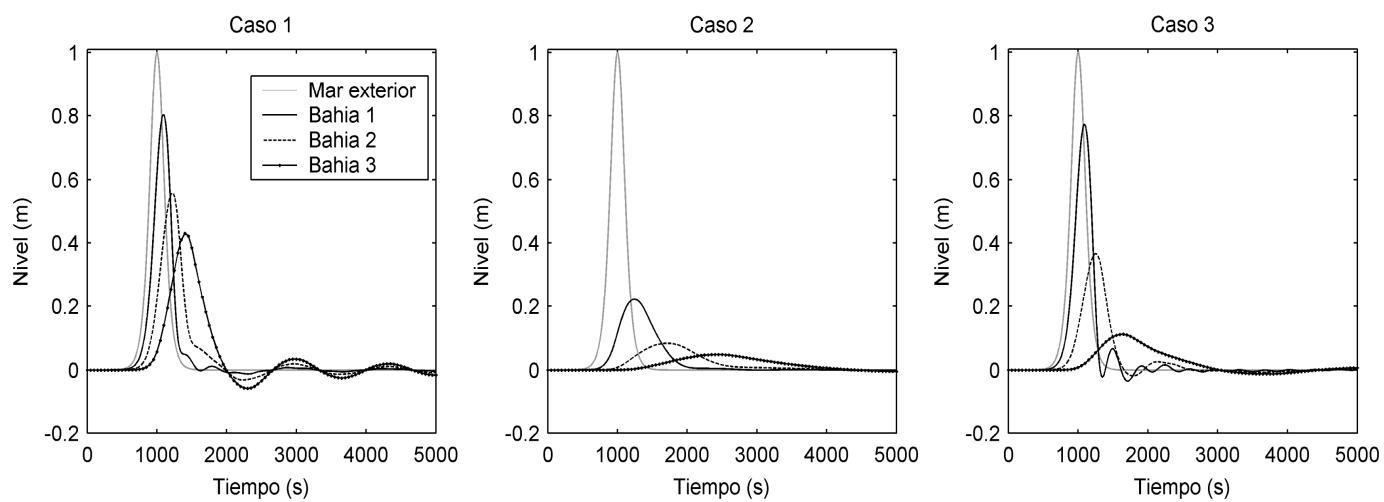

Figura 4. Solución del modelo para tres geometrías distintas en las que la longitud y profundidad de los canales se mantiene constante, $100 \mathrm{~m}$ de longitud y $10 \mathrm{~m}$ de profundidad. Caso 1: las tres bahías ocupan $10^{6} \mathrm{~m}^{2}$ y la anchura de los canales es de 200100 y $50 \mathrm{~m}$ respectivamente. Caso 2: geometría en la que los canales son más angostos, de 20, 10 y $5 \mathrm{~m}$ respectivamente. Caso 3 : la anchura de los canales es igual que en el caso 1 pero el tamaño de la bahía aumenta a medida que nos alejamos del mar, la bahía 1 ocupa $10^{6}$ $\mathrm{m}^{2}$, la bahía $2,5 \cdot 10^{6} \mathrm{~m}^{2}$ y la $3,5 \cdot 10^{6} \mathrm{~m}^{2}$

Como se puede observar, si los pasos de agua son más angostos (caso 2) la amplitud de la onda en cada una de las bahías será menor. El caso 3 muestra el efecto del tamaño de las bahías, si en lugar de ser todas las bahías iguales, como en el caso 1 , el tamaño de las mismas aumenta a medida que nos alejamos del mar exterior, la amplitud de la onda será menor porque el volumen de agua que pasa por el canal tiene que repartirse en un área mayor.

\section{VALIDACIÓN EXPERIMENTAL}

\section{Descripción del ensayo}

Los tsunamis son eventos esporádicos con una baja probabilidad de ocurrencia y por lo tanto, validar el modelo numérico con datos de campo resulta prácticamente imposible. Para solventar este problema, el modelo numérico agregado propuesto se ha validado con un ensayo de laboratorio realizado en el tanque de oleaje del laboratorio del Instituto de Hidráulica Ambiental "IH Cantabria", de la Universidad de Cantabria.

El tanque de oleaje tiene unas dimensiones de $28.4 \mathrm{~m}$ de longitud, $8.6 \mathrm{~m}$ de anchura y $1.2 \mathrm{~m}$ de altura. Está dotado de un sistema de generación de oleaje compuesto por 10 palas de tipo pistón de $80 \mathrm{~cm}$ de anchura cada una, accionadas mediante servomotores de corriente alterna, con una carrera máxima de $1 \mathrm{~m}$ y una potencia instalada de $100 \mathrm{~kW}$.

Aunque la aplicación fundamental de las palas del tanque es la generación de oleaje de corto periodo, el sistema de control así como la geometría de las palas (espacio entre ellas y dimensiones del faldón de cada pala), permiten la generación de ondas largas de hasta $20 \mathrm{~s}$ de período, con una altura de ola máxima de 5 a $10 \mathrm{~cm}$, según la profundidad. Asimismo, la potencia instalada permite la generación de ondas solitarias cuya altura puede llegar al $50 \%$ de la profundidad disponible frente a las palas.

El modelo físico realizado consiste en 3 bahías conectadas en serie. Las bahías 1 y 2 miden 1.5 $\mathrm{m} \times 1.5 \mathrm{~m}$ mientras que la laguna 3 tenía 1.5 $\mathrm{m}$ de anchura y $1.25 \mathrm{~m}$ de longitud. Las anchuras de la bocana son de 21.5, 11.5 y $5.5 \mathrm{~cm}$ respectivamente. El sistema se construyó en el centro del tanque, sobre una plataforma horizontal, que simulaba un fondo de profundidad constante, precedida de una pendiente a modo de plataforma continental. Así se representaban los efectos de asomeramiento de la onda al adentrarse en aguas someras, que es donde se localiza la estructura del ensayo (Figura 5).

Las oscilaciones de la superficie libre se registran con sensores de nivel tipo resistivos, previamente calibrados. Se colocó un sensor a $1 \mathrm{~m}$ de distancia de la bocana, para conocer la onda en el exterior de sistema (forzamiento), dos en la bahía 1 y uno en cada una de las bahías 2 y 3 (Figura 6).

\section{Resultados del modelo físico}

Los registros de nivel obtenidos en el ensayo de laboratorio, presentan oscilaciones de alta frecuencia asociadas a la reflexión con los contornos. Estas oscilaciones se han filtrado promediando los datos con una media móvil. 


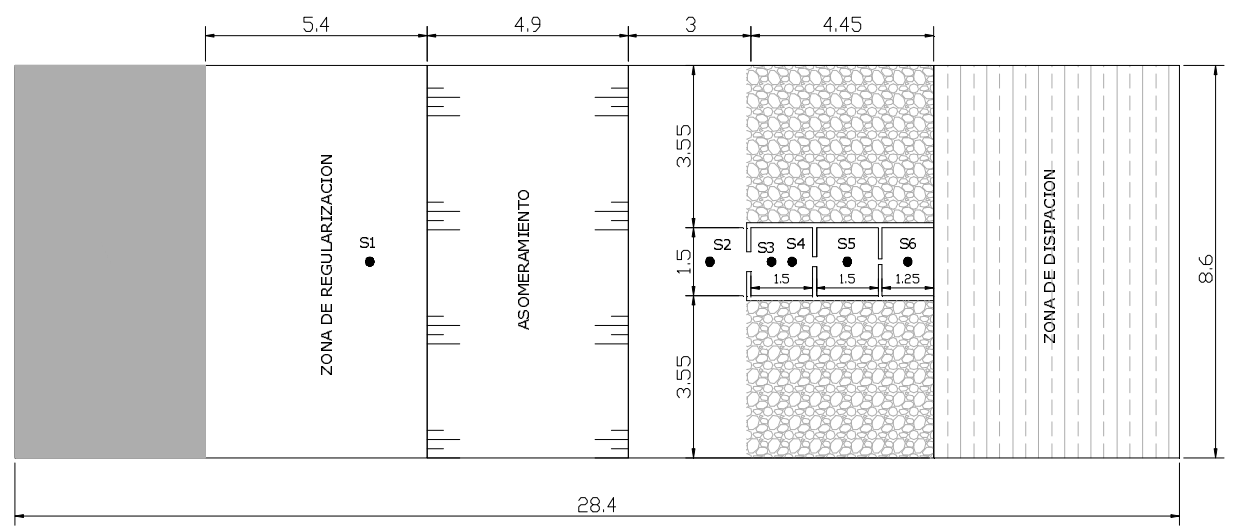

Figura 5. Esquema descriptivo del tanque y la configuración geométrica del ensayo

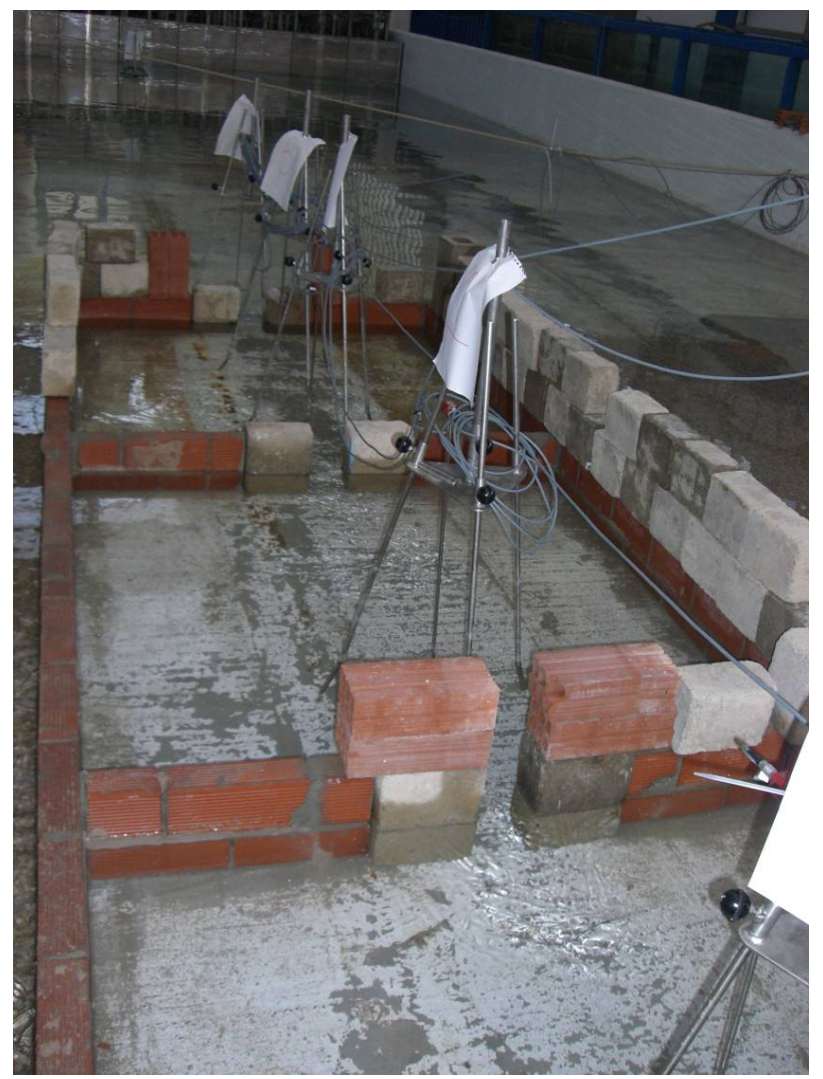

Figura 6. Fotografía del ensayo en la que se puede ver la ubicación de los sensores

En la Figura 7 se presentan las variaciones de nivel registradas en el ensayo. Como cabe esperar la amplitud del solitón inicial disminuye conforme alcanza los diferentes cuerpos de agua, porque el paso de agua es cada vez más estrecho a medida que nos alejamos del mar exterior. De este modo, la onda en el exterior tiene una altura de $2.13 \mathrm{~cm}$, en la bahía 1 ésta se reduce a $1.36 \mathrm{~cm}, 0.76 \mathrm{~cm}$ en la bahía 2 y $0.61 \mathrm{~cm}$ en la
3. También se puede apreciar un ligero retardo (o desfase) que se debe a la dificultad del flujo en atravesar el canal de comunicación con el cuerpo de agua semi-confinado.

Como se puede apreciar, las variaciones de nivel registradas en el exterior en los instantes posteriores a la generación del solitón también provocan una respuesta en el interior del sistema. 


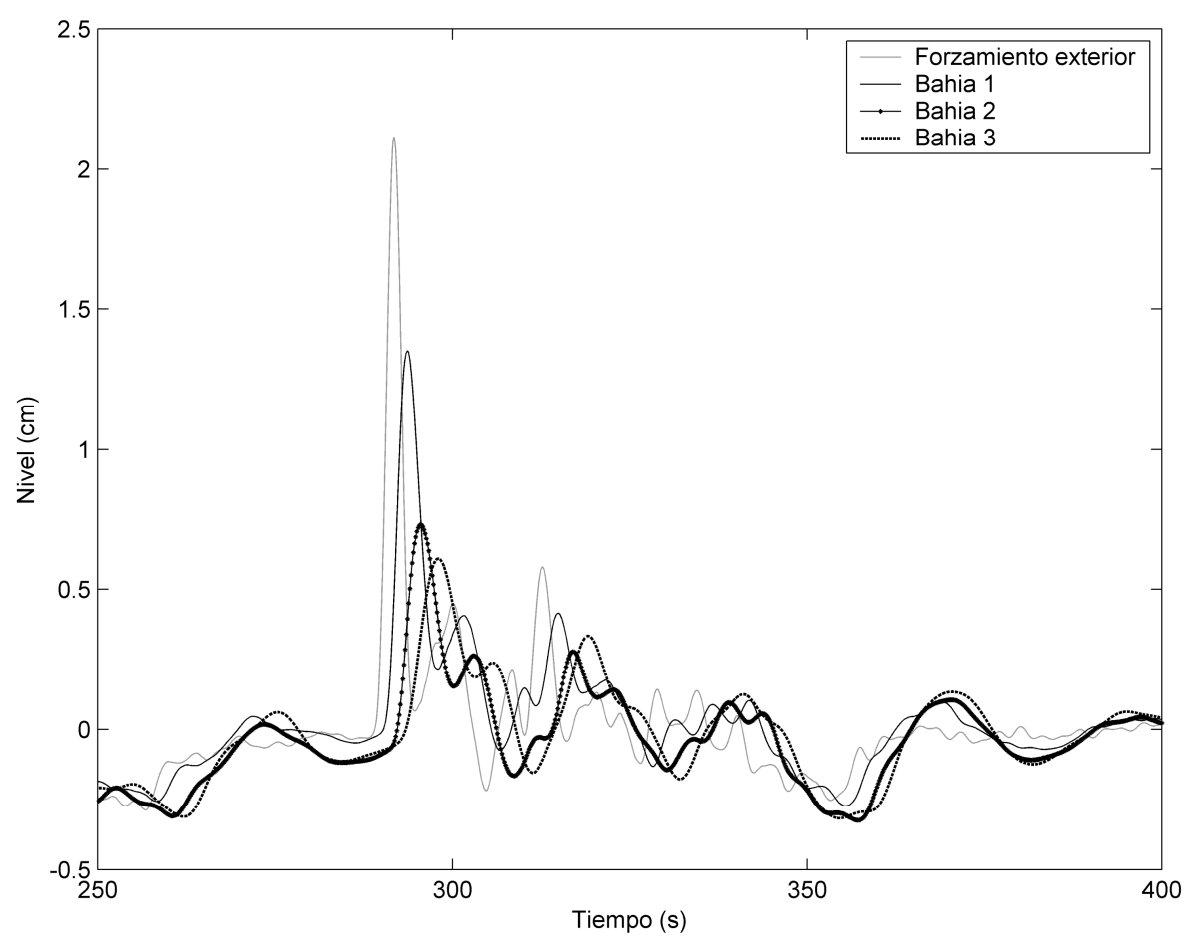

Figura 7. Variaciones de nivel registradas en el ensayo

\section{Validación del modelo numérico}

El parámetro que se utiliza para validar el modelo numérico es el que agrupa las pérdidas de carga localizadas a la entrada y a la salida del canal, $K$.

Puesto que se dispone de los registros obtenidos con el modelo físico, se ha realizado una validación por ajustes, que consiste en encontrar el valor de $K$ que consiga la mejor aproximación de la solución del modelo a los datos medidos. Para ello se calcula el error cuadrático medio, $\varepsilon_{r m s}$, de la serie registrada en el laboratorio, con la obtenida del modelo numérico en cada una de las bahías y se encuentra la combinación óptima de los parámetros $K_{1}, K_{2}, K_{3}$, que minimizan el error cuadrático medio. El menor $\varepsilon_{r m s}$ para la bahía 1 se encontró con valores de $K_{1}$ que oscilan entre 0.8 y 1 , para la bahía 2 , el rango es de 0.7 a 0.95 y para la tercera bahía entre 0.5 y 0.6 . La combinación del parámetro $K$ que consigue el $\varepsilon_{r m s}$ mínimo se consigue con $K_{1}=1, K_{2}=0.9$ y $K_{3}=0.55$. En la Figura 8 se muestra la comparación del registro obtenido en el laboratorio con la solución del modelo numérico, utilizando estos valores del parámetro $K$. El forzamiento exterior introducido en el modelo numérico es el registro medido en el exterior del estuario.

Teniendo en cuenta que generalmente no se dispone de datos con los que comparar la solución del modelo numérico, se procede a realizar una validación ciega, que consiste en comprobar cuál sería el resultado del modelo asumiendo que el valor del parámetro $K$, para cada uno de los pasos de agua, es 1.0, que es el valor típico que se utiliza en ingeniería para la propagación de ondas largas a través de secciones estranguladas (Blevins, 1992). En la Figura 9 se representa la solución del modelo numérico utilizando $K=1.0$ para todas las bahías y se compara con el registro de nivel obtenido en el laboratorio.

El error existente entre la serie medida y la modelada oscila entre un 3 y un $5 \%$ en ambos casos. Las diferencias en el error entre ambas validaciones son pequeñas, de modo que se puede asumir que el valor de calibración del parámetro $K$ es igual a 1.0, con la finalidad de utilizar el modelo numérico como método predictivo.

Cabe destacar cómo el modelo numérico reproduce, en cada una de las bahías, no sólo la llegada del solitón inicial sino también las posteriores variaciones de nivel, tanto positivas como negativas. Por tanto, se ha demostrado que el modelo numérico es una herramienta adecuada para conocer y describir el comportamiento hidrodinámico de un cuerpo de agua semiconfinado ante la llegada de un tsunami a la costa. 
Bahia $1 \varepsilon_{\mathrm{rms}}=0.042897 \mathrm{~cm}$
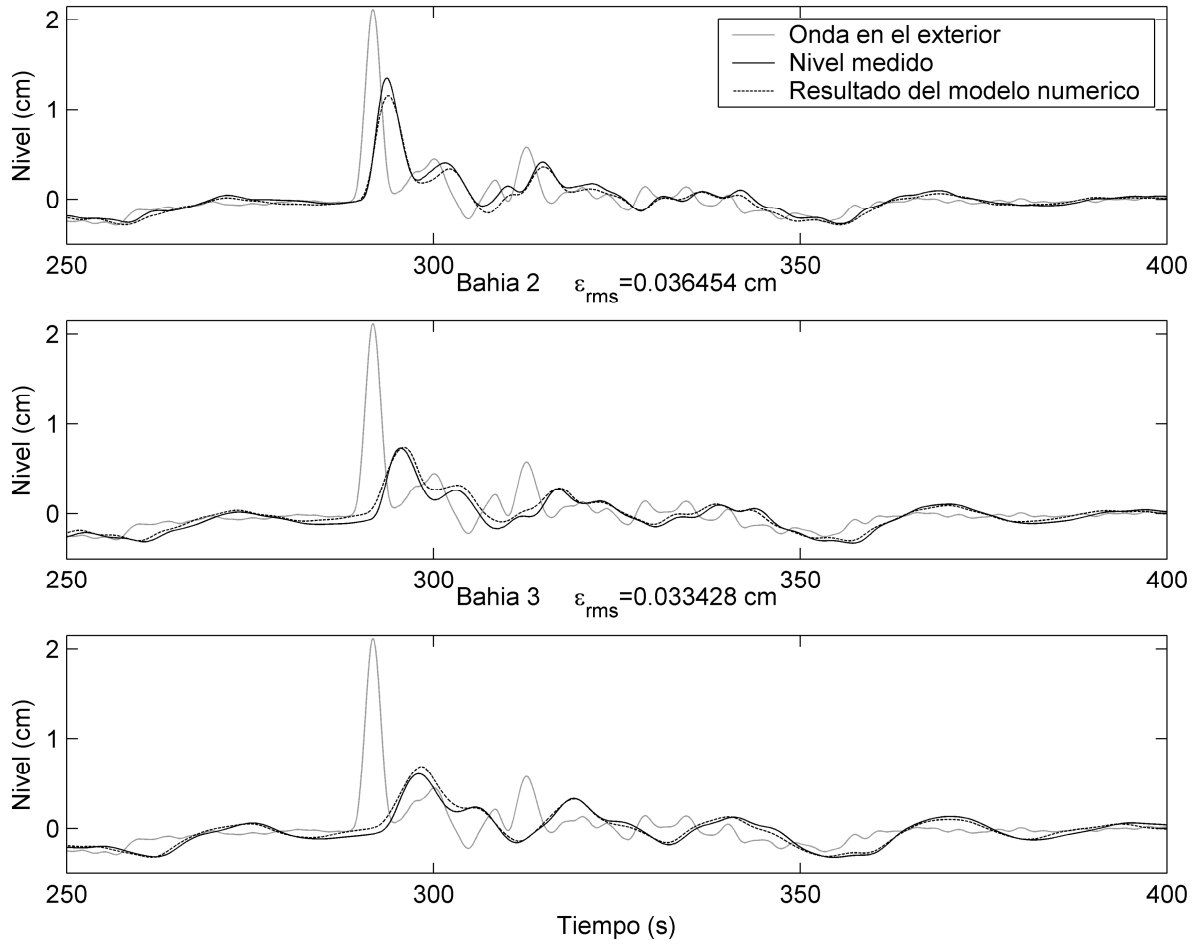

Figura 8. Comparación del nivel registrado en el estuario, para cada una de las bahías que lo conforman, como consecuencia de la propagación de una onda solitaria y el resultado obtenido con el modelo numérico para el caso de validación por ajustes $\left(K_{1}=1, K_{2}=0.9\right.$ y $\left.K_{3}=0.55\right)$

Bahia $1 \varepsilon_{\mathrm{rms}}=0.045212 \mathrm{~cm}$
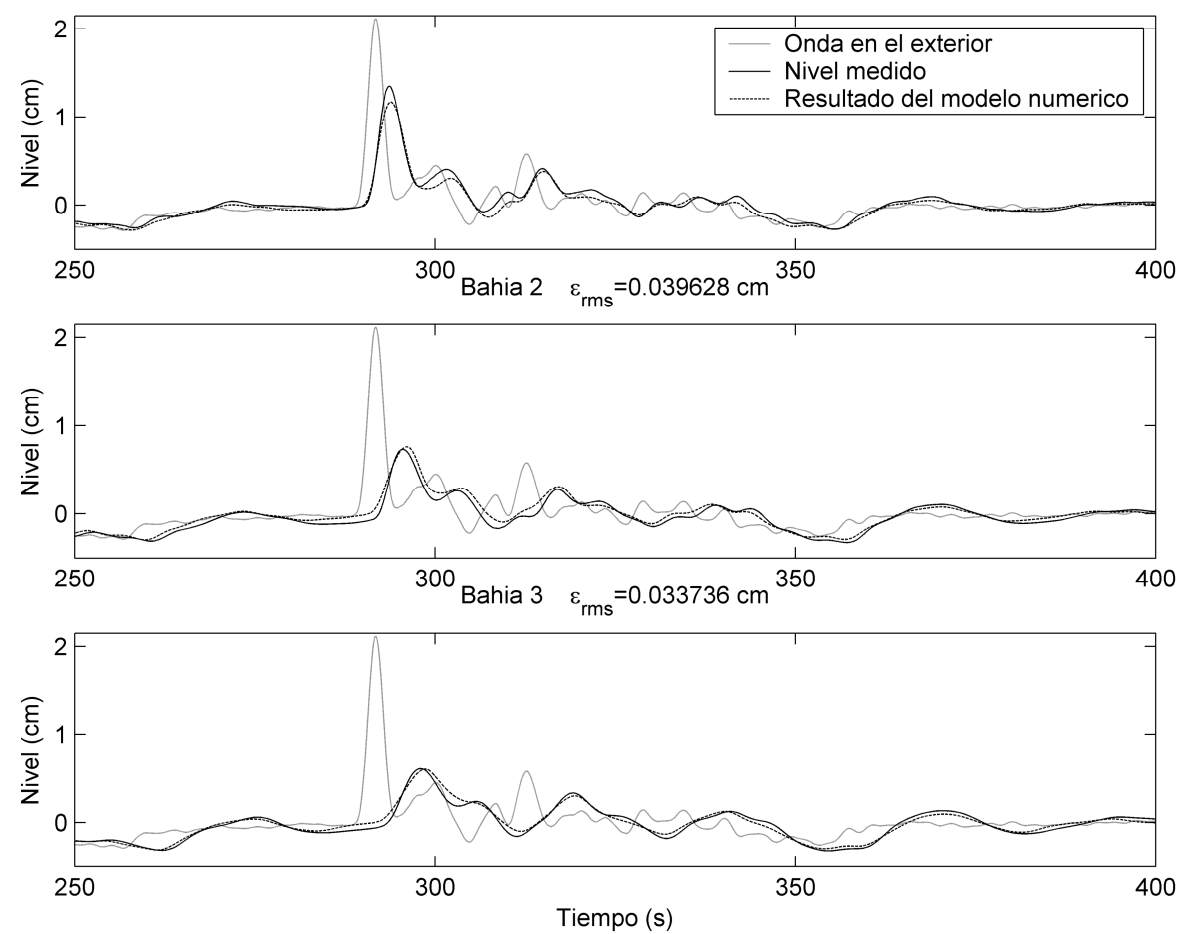

Figura 9. Comparación del nivel registrado en el estuario, para cada una de las bahías que lo conforman, como consecuencia de la propagación de una onda solitaria y el resultado obtenido con el modelo numérico para el caso de validaciones ciegas $\left(K_{1}=1, K_{2}=1\right.$ y $\left.K_{3}=1\right)$ 


\section{CONCLUSIONES}

En este artículo se presenta la aplicación de un modelo numérico agregado para conocer la respuesta de un tsunami en el interior de un cuerpo de agua semi-confinado. El modelo numérico desarrollado es aplicable a sistemas compuestos por más de una bahía y cuyos pasos de agua, tanto entre ellas como con el mar exterior sean angostos.

Para validar el modelo se ha realizado un modelo físico en laboratorio y la diferencia entre los resultados del modelo numérico y los datos obtenidos en el ensayo es inferior al $5 \%$, de modo, que se puede afirmar que el modelo numérico utilizado es una herramienta eficaz para conocer, en primera aproximación, la respuesta de un sistema costero ante la llegada de un tsunami.

Se propone la utilización de un sistema híbrido, en el que el modelo agregado descrito se acople con modelos numéricos avanzados de propagación de tsunamis, para reducir el esfuerzo computacional que se requiere en el caso de resolver la hidrodinámica de sistemas costeros semi-confinados utilizando mallas de detalle (con un gran número de celdas). Así, resolviendo con un modelo numérico avanzado la propagación de un tsunami hasta el exterior del cuerpo de agua de interés, el modelo agregado obtiene cómo serán las oscilaciones del nivel en el interior, en término medio.

El modelo numérico agregado es una herramienta que responde a las necesidades de la Directiva Europea relativa a la evaluación y gestión de los riesgos de inundación de zonas costeras.

\section{LISTA DE SÍMBOLOS}

$u$ : velocidad del flujo;

$g$ : aceleración de la gravedad;

$L$ : longitud del canal que comunica la bahía con el mar;

$\eta_{o}$ : nivel del mar en el exterior de la bahía;

$\eta_{b}$ : nivel del mar en el interior de la bahía;

$F$ : término de fricción;

$V$ : volumen de agua de la bahía;

$A_{c}$ : área de la sección del canal que comunica la bahía con el mar;

$K_{\text {ent }}$ : pérdida de carga localizada a la entrada;

$K_{s a l}$ : pérdida de carga localizada a la salida;

$f$ : término disipativo de Darcy-Weisbach;

$h_{c}$ : profundidad del canal correspondiente al nivel medio;
$A_{c}^{o}$ : área transversal del canal correspondiente al nivel medio;

$W_{c}$ : anchura del canal que comunica la bahía con el mar;

$A_{b}$ : área de la bahía o lagoon;

$H_{x}$ : altura de la onda solitaria;

$h_{x}$ : profundidad por debajo del nivel medio que hay en el exterior del sistema;

$c$ : celeridad de la onda solitaria;

$K$ : parámetro que agrupa las pérdidas de carga localizadas a la entrada y salida del canal;

$\varepsilon_{r m s}$ : error cuadrático medio.

\section{REFERENCIAS}

Blevins, R.D., (1992). Applied Fluid Mechanics Handbook. Krieger Publishing Company.

Bruun, P., Mehta, A.J. y Jonsson, I.G., (1978). Stability of Tidal Inlets: Theory and Engineering. Elsevier Scientific Publishing Co., Amsterdam, The Netherlands.

Defant A., (1961). Physical Oceanography, Vol. 1. New York, Pergamon, 598 pp.

Di Lorenzo, J.L., (1988). The overtide and filtering response of small inlet/bay systems. En Aubrey, D.G. y Weishar, L. (Eds.), Hydrodynamics and Sediment Dynamics of Tidal Inlets, Springer, New York, pp. 2453.

Friedrichs, C.T. y Aubrey, D.G., (1988). Nonlinear tidal distortion in shallow well-mixed estuaries: a synthesis. Estuarine, Coastal and Shelf Science, 27, 521-545.

Friedrichs, C.T. y Madsen, O.S., (1992). Nonlinear diffusion of the tidal signal in frictionally dominated embayments. J. Geophys. Res., 97 (C4), 5637-5650.

Jain, M., (2002). Hydraulics and stability of Multiple-Inlet bay systems: St. Andrew Bay, Florida. Thesis for the Degree of Master of Science, University of Florida.

Keulegan, G.H., (1951). Third progress report on tidal flow in entrances. Water level fluctuations of basins in communication with seas. Report 1146, National Bureau of Standards DC, 32 pp.

Maas, L.R.M., (1997). On the nonlinear Helmholtz response of almost-enclosed tidal basins with sloping bottoms. J. Fluid Mech., 349, 361-380.

Ridderinkhof, H., (1988). Tidal and residual flows in the western Dutch Wadden Sea: 
II. An analytical model to study the constant flow between connected tidal basins. Neth. J. Sea Res., 22(3), 185-198.

Salles, P., (2001). Hydrodynamic controls on multiple tidal inlet persistence. Ph.D. Thesis of Massachusetts Institute of Technology and Woods Hole Oceanographic Institution, $266 \mathrm{pp}$.

Speer, P.E. y Aubrey, D.G., (1985). A Study of non-linear tidal propagation in shallow inlet/estuarine systems. Part II: Theory. Estuarine, Coastal and Shelf Science, 21, 207-224.

Stanev, E.V., Flöser, G. y Wolff, J.O., (2003a). First- and higher-order dynamical controls on water exchanges between tidal basins and the open ocean. A case study for the East Frisian Wadden Sea. Ocean Dynamics, 53, 146-165.

Stanev, E.V., Burchard H., Bolding K. y Flöser, G., (2003b). On the circulation in the East Frisian Wadden Sea: numerical modelling and data analysis. Ocean Dynamics, 53, 27-51.

Van de Kreeke, J., (1988). Hydrodynamics of tidal inlets. Hydrodynamics and sediment dynamics of tidal inlets, coastal and estuarine studies 29. Edited by D.G. Aubrey and L. Weishar, Springer-Verlag, New York.

Van de Kreeke, J., (1990). Can multiple tidal inlets be stable? Estuar. Coast. Shelf Sci., $30,261-273$ 